Proc. Estonian Acad. Sci. Eng., 2006, 12, 1, 16-25

\title{
Rough surface model using potential function superpositions
}

\author{
Tanel Telliskivi ${ }^{\mathrm{a}}$ and Priit Põdra ${ }^{\mathrm{b}}$ \\ a Department of Machine Design, Royal Institute of Technology, Samaritgr. 7, 11853 Stockholm, \\ Sweden; tanel@kth.se \\ b Department of Mechatronics, Tallinn University of Technology, Ehitajate tee 5, 19086 Tallinn, \\ Estonia; priitp@staff.ttu.ee
}

Received 18 June 2004, in revised form 20 June 2005

\begin{abstract}
A fast method of contact mechanics for rough surfaces is presented. The roughness beneath each contact element of the discretized contact area is described by a single asperity, having the shape of the Abbott Firestone Curve and the modified carrying stiffness for rough contact is modelled with the help of bars. The model was verified by solving several example problems, showing high computing speed. The number of elements can be changed easily in contact area and the repetitiveness of the method for a rough surface is demonstrated.
\end{abstract}

Key words: Hertz problem, rough surface, normal indentation, potential function, Abbott Firestone Curve.

\section{INTRODUCTION}

The microgeometry of surfaces is critical to the success of most manufacturing processes and to the performance of manufactured products. The application of the Abbott Firestone Curve (AFC) along with the contact mechanics method that uses the potential function superpositions (PFS) is combined in order to simulate many kinds of engineering contacts. The contact area has been divided into rectangular elements with the constant pressure of Love $\left.{ }^{1}\right]$ and respective potential function solutions are used in a superposition method for the unknown shape of the contact area $\left[^{2}\right]$. This approach uses the so-called half-space assumption stating that the neighbouring elements should not have dissimilarities in height. If they exist, the magnitude of pressure between the elements becomes unrealistically high. Similar problems have been 
solved before using the matrix inversion method $\left[^{3}\right]$ and the variational method $\left[{ }^{4}\right]$ for arbitrary shapes of contacting objects. The last two methods use an outer loop of iterations to enforce the equilibrium constraint, based on the boundary conditions. In the present method the bodies are brought into contact stepwise with the load being applied in increments permitting the adjustment of stiffness, when needed, straight from the initial point contact. The height of a flat element can be adjusted, minimizing the height difference of the elements for PFS computation. The real surface beneath the element is irregular and an additional modelling is carried out. The element stiffness is adjusted by adding a bar, which has a stiffness and an approximate contacting area, determined with the help of AFC analysis.

Implementation of the AFC has been originally done for a rigid foundation $\left[{ }^{5}\right]$. In $\left[^{6,7}\right]$ this implementation was applied together with the Finite Element (FE) wear modelling. The FE method provides material penetration due to the normal load and the modelling of surface roughness can be separated as it is made in the present work. It is known that solving contact problems for a rough surface using the potential functions, which are based on the linear elasticity theory, the resulting maximum pressure value is by far overestimated as compared to the experiments, especially with steels. The real contact parameters have been determined using FE analysis $\left[{ }^{8}\right]$ or modelling the softening, depending on the roughness properties. Rough surfaces can be handled in such a way that the stiffness of each contacting element can be "adjusted" at the start of the incremental contact solution (specific for PFS). The contact area and the contact pressure distribution of real contacts have been measured with an ultrasonic method $\left[{ }^{9}\right]$ and may be a way of calibrating the presented method.

\section{THEORY FOR THE PFS MODEL}

Consider two interacting bodies in a Cartesian coordinate system with the origin chosen approximately in the centre of the contact locality. The contact area is a rectangle (surface matrix) that is large enough to cover the true contact region with edges parallel to the $x$ and $y$ axes in the $x y$ plane and is divided into $m \times n$ rectangular elements with boundaries $2 \xi \times 2 \eta$. The $z$ axis points vertically upwards into the upper body.

\subsection{Uniform pressure distribution on the subdivision area}

Well-known influence coefficient functions, based on the half-space assumption, are used to determine the relation between the displacement $u_{z}$ and the pressure $p$. Each rectangular element is subjected to uniform stress distribution, with pressure in the normal direction. The following equation is used: 


$$
\begin{aligned}
\frac{2 \pi G u_{z}}{(1-v) p}=C(x, y)= & (\eta+y) \log \left(\frac{\xi+x+\sqrt{(\xi+x)^{2}+(\eta+y)^{2}}}{-\xi+x+\sqrt{(x-\xi)^{2}+(\eta+y)^{2}}}\right) \\
& +(y-\eta) \log \left(\frac{\xi+x+\sqrt{(\xi+x)^{2}+(y-\eta)^{2}}}{-\xi+x+\sqrt{(x-\xi)^{2}+(y-\eta)^{2}}}\right) \\
& +(\xi+x) \log \left(\frac{\eta+y+\sqrt{(\xi+x)^{2}+(\eta+y)^{2}}}{-\eta+y+\sqrt{(\xi+x)^{2}+(y-\eta)^{2}}}\right) \\
& +(x-\xi) \log \left(\frac{\eta+y+\sqrt{(x-\xi)^{2}+(\eta+y)^{2}}}{-\eta+y+\sqrt{(x-\xi)^{2}+(y-\eta)^{2}}}\right),
\end{aligned}
$$

where $G$ is the shear modulus and $v$ is the Poisson ratio. Equation (1) shows the relation between displacement $u_{z}$ and pressure $p$ for a rectangular contact patch with an element area $A=2 \eta \times 2 \xi$, centred at the origin of the coordinate system and subjected to a uniform pressure.

The logarithmic function $C(x, y)$ converts the constant pressure over a rectangular area to the deformation field $u_{z}$ (in this direction only) within the bounds $-\infty$ and $\infty$ and forms a matrix $\mathbf{u}_{z}$ over the contact area. Discrete form of $C(x, y)$ is denoted by the matrix $\mathbf{C}$. Matrix $\mathbf{C}$ is real-valued and symmetric. The matrix $\mathbf{C}$ has originally dimensions $(2 m+1) \times(2 n+1)$ and is then reduced to the size $m \times n$ at the proper indices as illustrated in Fig. 1 and then used for the direct superposition at the contact locality.

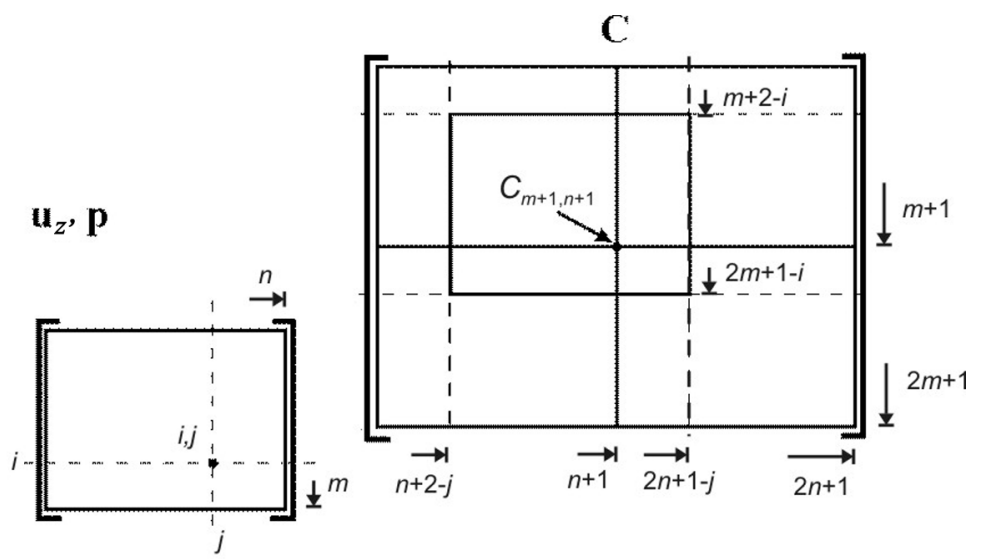

Fig. 1. Fit of the matrix $\mathbf{C}$ for the incremental computation point. 


\subsection{Half-space solution}

In the proposed PFS method, the original shape (no inversion) of $C(x, y)$ is used, operating with what are called scaled displacements, because the pressure distribution $\mathbf{p}$ is not known beforehand. The distances between different $u_{z i, j}$ components are constant. The following steps are required.

1. The position matrices $\mathbf{x}$ and $\mathbf{y}$ are created in order to specify the origin of the coordinate system for a number of matrices, among others $\mathbf{u}_{z}$, $\mathbf{p}$ etc.

2. At the start the matrix $\mathbf{u}_{z}=0$.

3. The increment step size $\Delta \delta_{d i, d j}$ is defined for the highest point of overlap, indexed with combined indices $d i, d j$, where $d$ is the queue number of the step and $i$ or $j$ is the position of the element in the contact locality; $d, i$ and $j$ are used in combination because their magnitude is unique and depends on the form of the rough surface. The increment step size can be accumulated into the $m \times n$ matrix $\Delta \delta$, where the single increment step size $\Delta \delta_{d i, d j}$ is concatenated with the indices $d i, d j$.

4. The single increment step size $\Delta \delta_{d i, d j}$ has a determined magnitude, usually a fraction of the overlap. The term overlap means the subtraction of the upper body from the lower achieving the points of matrix above the zero level, which makes the basis for the contact computation.

If the load at the given approach is to be found, the upper body is first shifted by the size of approach to ensure positive values of the overlap. The single increment step with the influence matrix modifies the geometry (displacement field $\mathbf{u}_{z}$ ). For every increment step size $\Delta \delta_{d i, d j}$ in its specific location the adaptation of $\mathbf{u}_{z}$ is performed as follows:

$$
\mathbf{u}_{z}=\sum_{d}\left(\left[\begin{array}{cccc}
C_{m+2-d i, n+2-d j} & \ldots & & C_{m+2-d i, 2 n+1-d j} \\
\ldots & \ldots & & \ldots \\
& & & \\
C_{2 m+1-d i, \mathrm{n}+2-d j} & & \ldots & C_{2 m+1-d i, 2 n+1-d j}
\end{array}\right] \cdot \frac{\Delta \delta_{d i, d j}}{C_{m+1, n+1}}\right) .
$$

In the same way as in Eq. (2), the matrices are subtracted cumulatively from the overlap. Locations beside the increment steps are affected by the influence function and decrease rapidly in the far field by the scaling of the length $\Delta \delta_{d i, d j}$ logarithmically, as in Eq. (2).

The total displacement field $\mathbf{u}_{z}$ increases cumulatively due to this neighbouring cell impact for every $\Delta \delta_{d i, d j}$ :

$$
P_{d i, d j}=\frac{-\pi G \Delta \delta_{d i, d j}}{C_{m+1, n+1}(v-1)} \Delta x \Delta y,
$$

where $\Delta x=2 \xi$ and $\Delta y=2 \eta$. The increment steps $\Delta \delta_{d i, d j}$ that are fractions of the local maximum of the subtracted height matrices of the two bodies can be 
converted linearly to the force as presented in Eq. (3). This is valid for the linearelastic material model.

If the approach of the two bodies at the load $P$ is to be found, the shift of the upper body is taken approximately and the condition $\Sigma(\mathbf{p} \cdot \Delta x \cdot \Delta y) \geq P$ stops the calculation.

\section{MODELLING INFLUENCE OF THE SURFACE ROUGHNESS ON CONTACT PRESSURE}

Surface topography in the microscale is normally quite stochastic and statistical methods are needed to determine the parameters for engineering applications. The material distribution of a rough surface can be described by the "bearing area curve", AFC. As seen in Fig. 2a, every element of the rough surface has different height. In every element additional roughness exists. An additional calculation procedure for the sorted asperities, representing the AFC in three dimensions, is added. The model can be only two-dimensional (single-trace), because the asperities are relatively constantly spread in the $y$ direction (Fig. 2b). Higher asperities under a single element come into the contact earlier and create the contact areas $A_{d i, d j}$, consisting of a number of asperities. It is assumed that every asperity has the same cross-section. When an incremental approach of the two bodies develops, the force $P_{d i, d j}$ of one element contracts also the asperities (bars) inside the element by

$$
\Delta \delta_{d i, d j \mathrm{AFC}}=\frac{P_{d i, d j} L_{d i, d j}}{E A_{d i, d j}},
$$

where the displacement $\Delta \delta_{d i, d j \mathrm{AFC}}$ is added to $\Delta \delta_{d i, d j}$ of the half-space solution, $A_{d i, d j}$ is the effective contact area starting from the depth $h_{0}$ of the size $A_{0}$

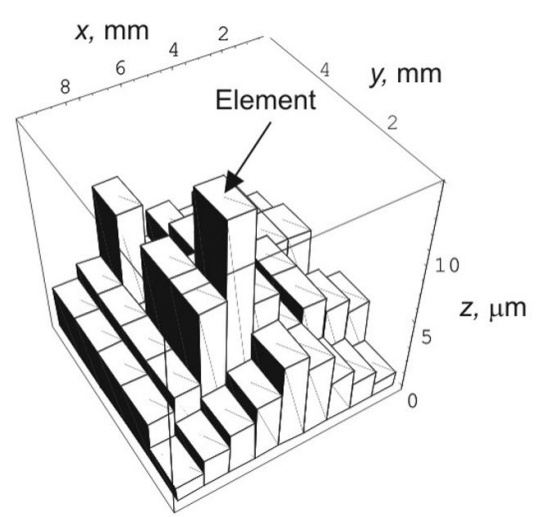

(a)

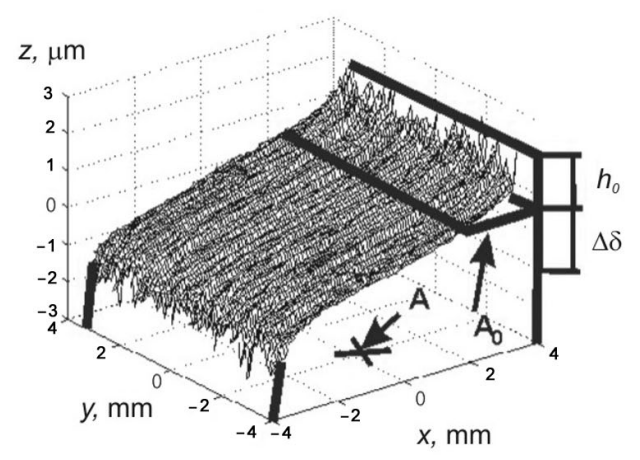

(b)

Fig. 2. Model for pressure and optionally for a rough surface, where the nodes of interest are in the centre of columns (a) and sorted asperities of an element (b). 
(Fig. 2b), $L_{d i, d j}$ is the length of the bar at the particular approach, $E$ is the modulus of elasticity and $P_{d i, d j}$ is the load on the particular element in the actual solution step. The length matrix $\mathbf{L}$ is created assuming Gaussian distribution of the asperities. According to the statistical methods, the length $L_{i, j}$ is three times the standard deviation of the rough field under the element. During the progress of the approach, $L_{i, j}$ is shortened to $L_{d i, d j}$ due to the compression.

\section{EXAMPLES}

This simple method, validated in this section, offers solutions to various threedimensional problems. When two rough elastic bodies are pressed against each other, the initial point of contact develops into the area of contact, due to the elastic deformation. A three-dimensional state of stress is induced in both bodies. The shape of the contact area was originally established by Hertz, who assumed that the contact takes place between second-degree surfaces. In this case the contact area is an ellipse (with major half-diameter $b$ and the minor half-diameter $a$ ). The Hertzian solution is given in $\left[{ }^{10}\right]$. The curvatures radii are calculated on the basis of the geometrical form of the contacting bodies.

\subsection{Comparison of smooth and rough surfaces using existing methods}

The contact of a cylinder and barrel under a load of $1600 \mathrm{~N}$ is analysed in Table 1. This contact is typical for two rollers. Radii $0.035 \mathrm{~m}$ and the radius of transversal curvature of the upper body $0.125 \mathrm{~m}$ was used.

The results with the smooth surface are summarized in Table 1, rows 1 and 2 . Here the comparison is made between the Hertzian method and the PFS method. The undeformed contacting geometries are shown in Fig. 3a. Deformed geometries are shown in Fig. $3 \mathrm{~b}$ and surface deformations and pressure in Fig. 4. Figures 3 and 4 are created using the PFS method. A solution for irregular surface by PFS is presented in Table 1 (row 3) and in Figs. 5 and 6. The roughness was added to the smooth geometries. The standard deviation of the roughness had a base magnitude of $0.01 \cdot \Delta x$, which was an input for the generation of the rough surface. Generation of the rough surface is described in $\left[{ }^{3}\right]$. The surface roughness causes very high value of the maximum pressure (Table 1 ).

Table 1. Comparison of the smooth geometry and rough surface

\begin{tabular}{lcccc}
\hline & Hertz method & Smooth surface & Rough surface \\
\hline Normal load, N & 1600 & 1600 & 1600 \\
Max pressure, MPa & 1406 & 1397 & 3308 \\
$2 b, \mathrm{~m}$ & $2.88 \cdot 10^{-3}$ & $2.94 \cdot 10^{-3}$ & $2.67 \cdot 10^{-3}$ \\
$2 a, \mathrm{~m}$ & $7.56 \cdot 10^{-4}$ & $7.78 \cdot 10^{-4}$ & $7.78 \cdot 10^{-4}$ \\
$\mathrm{a} / \mathrm{b}$ & 0.2625 & 0.2642 & 0.2917 \\
$\mathrm{~b} / \mathrm{a}$ & 3.81 & 3.79 & 3.43 \\
Approach, m & $1.27 \cdot 10^{-5}$ & $1.27 \cdot 10^{-5}$ & $1.33 \cdot 10^{-5}$
\end{tabular}




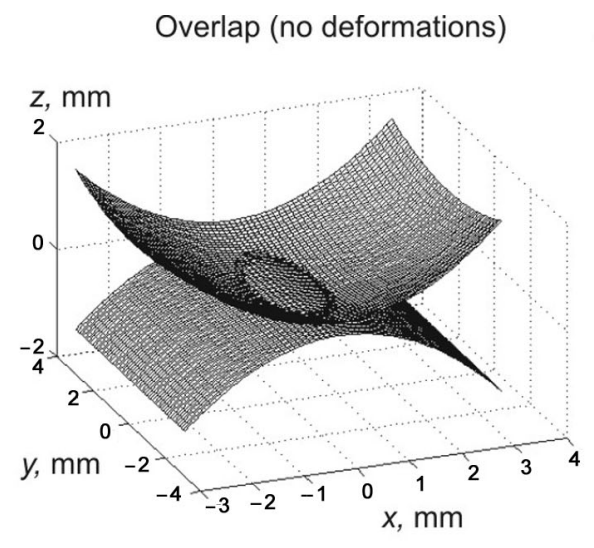

(a)

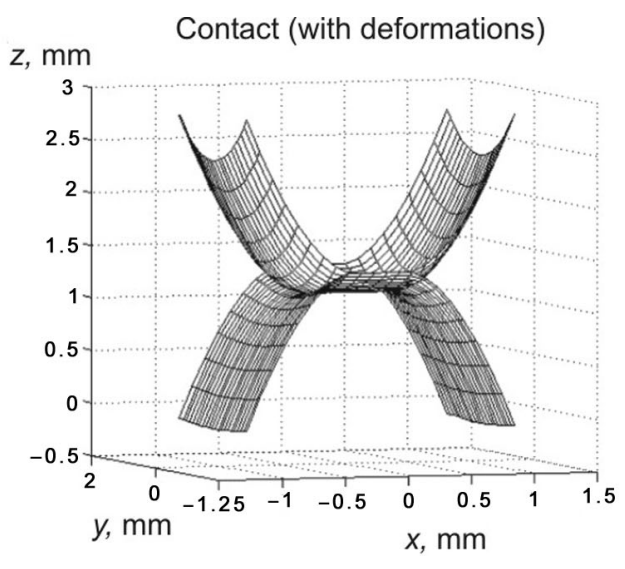

(b)

Fig. 3. Two discs in rigid interference (a) and elastic contact (b).

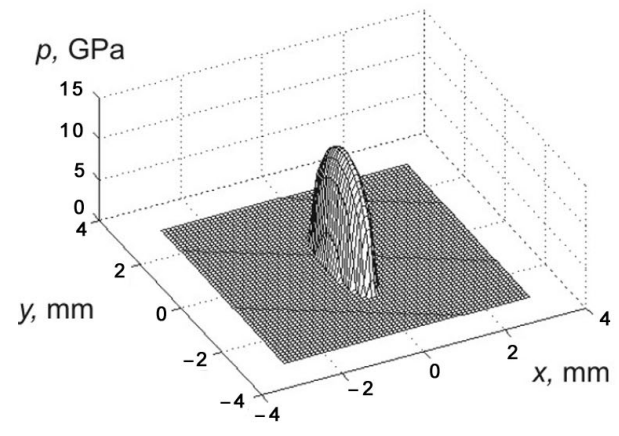

(a)

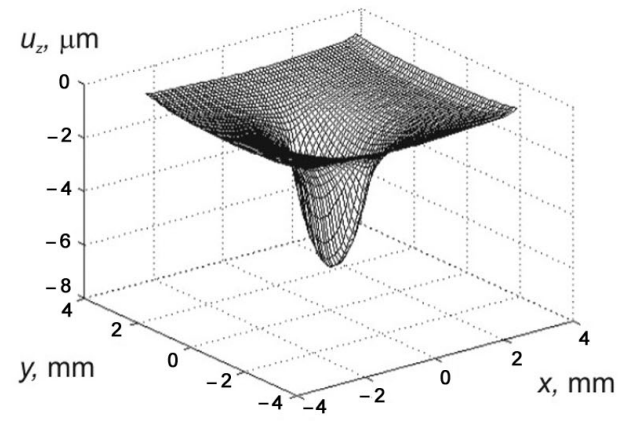

(b)

Fig. 4. Elastic pressure (a) and total elastic surface displacement of the smooth body (b).

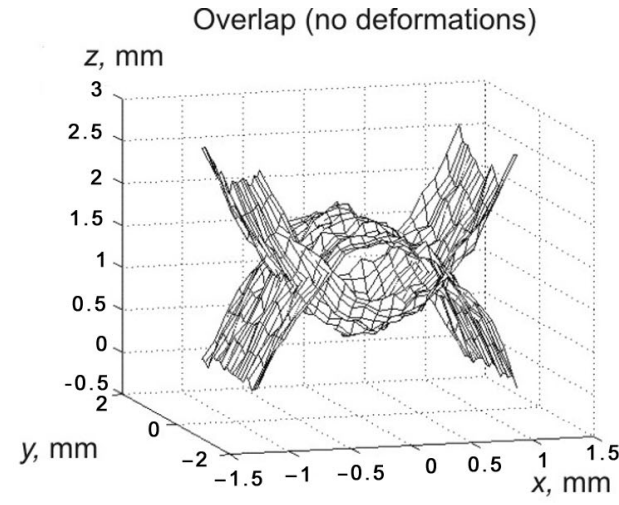

(a)
Contact (with deformations)

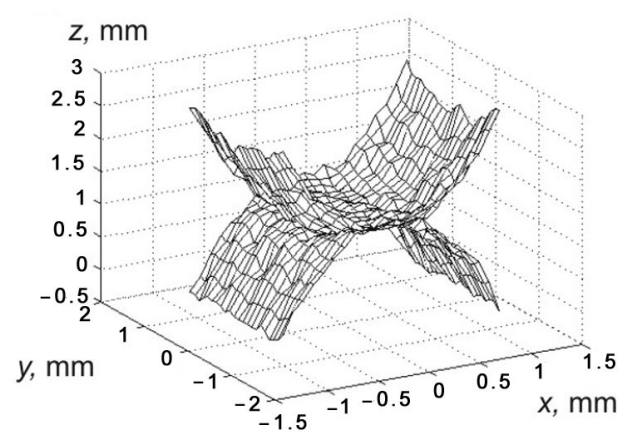

(b)

Fig. 5. Two rough discs in the rigid interference (a) and in the PFS elastic contact (b). 


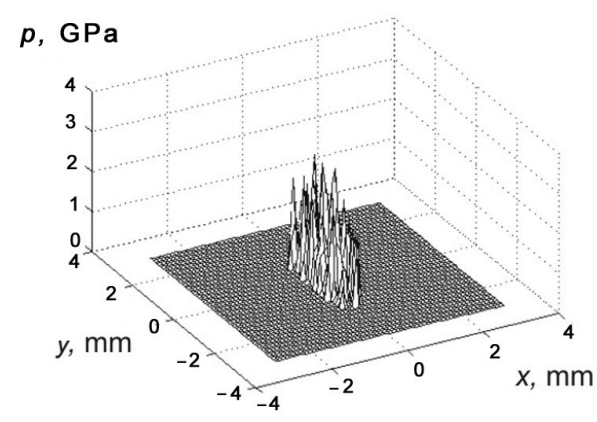

(a)

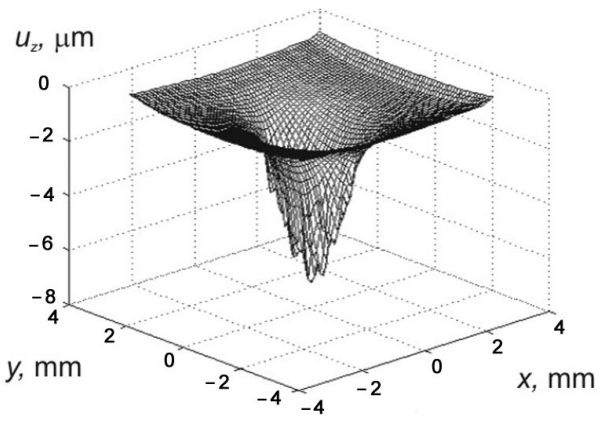

(b)

Fig. 6. Elastic pressure (a) and elastic surface displacement of the rough body (b) using solely PFS.

\subsection{Analysis of the proposed rough surface model}

Common way to analyse rough or worn parts starts with the surface measurement. Surface profilometers measure and record the roughness of a surface using either stylus or an optical beam. Typically, profilometers provide a choice of built-in electronic filters. Several thousand samples per centimetre can be measured. The presented method permits a variation in the amount of elements in the contact area, where the optimum is around $100 \times 100$ elements and might reach $500 \times 500$ elements. The elements represent the characteristic height for the rough rectangular area. For each element of the discretized contact area the mean value of many measurement points is assigned. The profilometers micrometer-sized diamond stylus tip acts as a mechanical low-pass filter and thus the raw profile is already smoothed to a certain extent prior to electronic filtering and also prior to any kind of numerical analysis. For instance, measurements with $1 \mathrm{~nm}$ step give 10000 samples per element for the AFC analysis when the contact is $1 \mathrm{~mm}$ long (in $x$ direction) and divided into 100 elements.

Two main conclusions can be drawn based on the results presented above. First, Fig. 7 demonstrates repetitiveness of the pressure contour patterns when changing the number of elements in the contact region.

Second, the half-space assumption of the PFS method is not violated any more, when the roughness is modelled with bars. Figure 8 shows the distribution of the contact pressure when the AFC, modelled with bars, is added to the PFS method. The number of elements in contact has risen from 210 to 300 resulting in the lowering of the maximum pressure from 3.3 (Table 1) to 2.8 GPa. The contour is not visually enlarged but the "islands" are often grown together. 


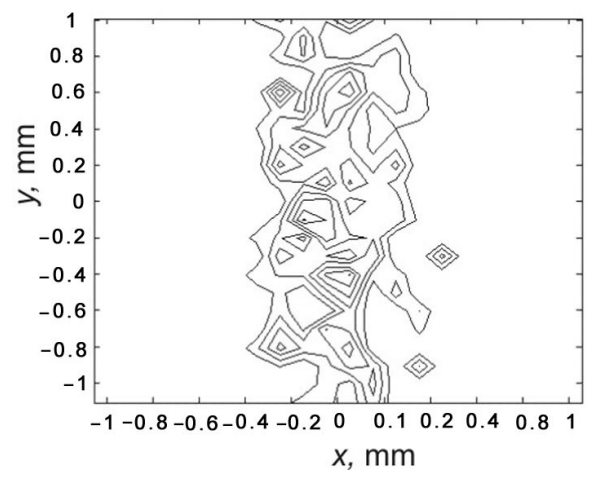

(a)

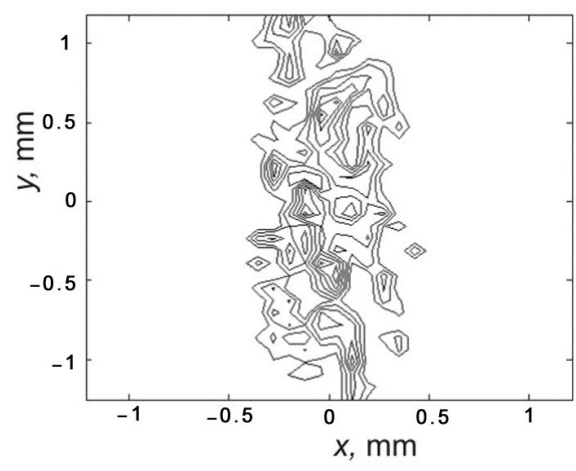

(b)

Fig. 7. Contour plots of the contact pressure, obtained with the PFS method: (a) $49 \times 49$ elements; (b) $91 \times 91$ elements.

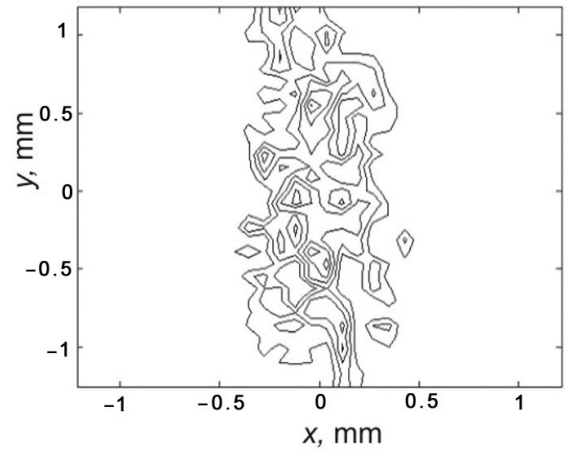

(a)

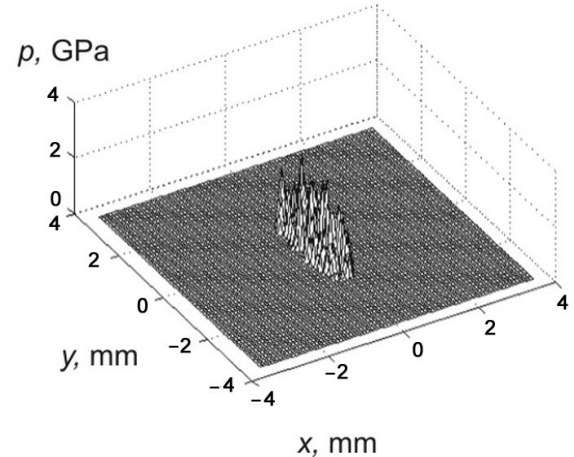

(b)

Fig. 8. Contact pressure distribution obtained applying PFS with AFC modelling, $91 \times 91$ elements.

\section{CONCLUSIONS}

Implementation of AFC along with the approximate contact mechanics calculation method PFS is described. The potential function solutions for rectangular contact elements under uniform pressure are used in the PFS method for arbitrary (non-elliptical) shapes of the contact area. The additional model above the PFS, consisting of single bars on PFS elements is characterized. The bar is created on the basis of AFC. The benefit of this method is that the contacting surfaces are not restricted by the limitations associated with the Hertzian contact. These limitations (half-space assumption etc.) are also valid for PFS. A complete solution for the elastic rough surface is proposed.

The repetitiveness of the pressure distribution with different numbers of the elements has been demonstrated. The bigger the element the more measurement 
samples can be included in the AFC analysis. The contour pattern of the contact pressure in the combined method remains complicated but looks very similar to the pressure distribution produced solely by the PFS method. The next step is to model plasticity of a rough surface in a similar manner. Plasticity under the surface becomes more and more dominant when increasing the load. When looking for the "true contact area" and for the "true pressure distribution", all of these models should work together.

\section{REFERENCES}

1. Love, A. E. H. The stress produced in a semi-infinite solid by pressure on part of the boundary. Phil. Trans. Royal Soc., A228, 1929, 377, 54-55.

2. Telliskivi, T. Wheel-Rail Interaction Analysis. PhD. Thesis. KTH, Stockholm, 2003.

3. Björklund, S. Elastic Contacts Between Rough Surfaces. PhD. Thesis, KTH, Stockholm, 1995.

4. Kalker, J. J. On the Rolling Contact of Two Elastic Bodies in the Presence of Dry Friction. $\mathrm{PhD}$. Thesis, Delft University of Technology, Delft, 1967.

5. Abbott, E. and Firestone, F. A. Specifying surface quality. Mech. Eng., 1933, 55, 569-572.

6. Põdra, P. and Andersson, S. FEA wear simulation of a conical spinning contact considering surface topography. Wear, 1997, 213, 13-21.

7. Sellgren, U., Björklund, S. and Andersson, S. A finite element-based model of normal contact between rough surfaces. Wear, 2003, 254, 1180-1188.

8. Telliskivi, T. and Olofsson, U. Contact mechanics analysis of measured wheel-rail profiles using the finite element method. J. Rail Rapid Transit, 2000, 215, 65-72.

9. Marshall, M., Lewis, R., Dwyer-Joyce, R., Olofsson, U. and Björklund, S. Ultrasonic characterisation of a wheel contact. In Proc. 30th Leeds-Lyon Symposium on Tribology. Lyon, 2003. CD-ROM.

10. Mägi, M. Versatile algorithms for exact computations of ovalities of Hertzian point contacts. In Matlab Conference. Stockholm, 1995. CD-ROM.

\section{Mõjufunktsioonide liitmistel põhinev kareda pinna mudel}

\section{Tanel Telliskivi ja Priit Põdra}

On esitatud ligikaudne ekspressmeetod karedate pindade kontaktmehaanika analüüsimiseks. Karedate pindadega kehade mehaanilise kontakti parameetrite määramisel on pinnakihtide jäikusel sisekihtide omaga võrreldes oluliselt suurem osatähtsus. Pinnakareduse modelleerimisel asendatakse siin diskretiseeritud kontaktala igale elemendile vastav tegelik pinnakaredus ühe summaarse pinnakonarusega, mille kuju vastab selle elemendi pinnatopograafia Abbotti kõverale. Selle põhjal kujundatakse iga pinnaelemendi jäikus. Meetodi usaldusväärsust on tõestatud mitme näidisülesande lahendamisega. Meetodi peamisteks eelisteks on arvutuse kiirus ja võimalus kontaktala elementide arvu hõlpsasti muuta. See teeb tulemused jälgitavaks mudeli rakendamisel nii siledate kui ka karedate pindade kontaktmehaanika modelleerimisel. 